\title{
$K_{1,1, m} \square P_{n}$ 的交叉数
}

\author{
欧阳章东 ${ }^{11 *}$ ，王晶 ${ }^{(2)}$, 黄元秋 ${ }^{3}$ \\ (1) 湖南第一师范学院数学系, 长沙 410205 ; \\ (2) 长沙学院信息与计算科学系, 长沙 410003; \\ (3) 湖南师范大学数学系, 长沙 410081 \\ E-mail: oymath@163.com, wangjing1001@hotmail.com, hyqq@hunnu.edu.cn
}

收稿日期: 2013-09-01；接受日期: 2014-08-20；＊通信作者

国家自然科学基金 (批准号: 11301169 和 11371133)、湖南省教育厅青年基金 (批准号: 12B026)、湖南省自然科学基金 (批准号:

$13 J J 4110$ 和 $14 J J 3138)$ 和湖南省高校科技创新团队支持计划资助项目

摘要 借助拉链积运算, Cartesian 积图 $K_{1, m} \square P_{n}$ 和 $K_{2, m} \square P_{n}$ 的交叉数最近被先后确定. 本文进一 步证明了: 对于 $m, n \geqslant 1$, 有

$$
\operatorname{cr}\left(K_{1,1, m} \square P_{n}\right)=2 n\left\lfloor\frac{m}{2}\right\rfloor\left\lfloor\frac{m-1}{2}\right\rfloor+(n-1)\left\lfloor\frac{m}{2}\right\rfloor .
$$

结论的证明基于 Bokal 关于树的 Cartesian 积图交叉数的有关结果. 另外, 我们也给出了确定 $K_{2, m} \square P_{n}$ 交叉数的一个简洁方法.

关键词 交叉数 Cartesian 积 拉链积 路

MSC (2010) 主题分类 $05 \mathrm{C} 10,05 \mathrm{C} 62$

\section{1 引言}

本文未加说明的图论术语均同文献 [1]. 图 $G=(V, E)$ 在平面上的一个画法, 是指图 $G$ 到平面 上的一个同胚映射 $\phi$, 其中, 图 $G$ 的每个顶点对应到平面上不同的节点, 图 $G$ 的边 $u v \in E$ 对应到 一条连接 $\phi(u)$ 和 $\phi(v)$ 的不穿过其他节点的连续弧. 在不引起歧义的情况下, 也称节点为顶点, 称弧 为边. 为了简单起见, 一个画法还应满足如下条件: (1) 没有三条边相交于一个公共的点, (2) 如果 两条边有一个公共的内部点 $p$, 则它们在点 $p$ 产生交叉, (3) 任意两条边只有有限个交叉点. 图 $G$ 的交叉数, 记作 $\operatorname{cr}(G)$, 是指在图 $G$ 的所有画法中边与边产生的交叉的最小值. 设 $\phi$ 是图 $G$ 的一个 画法, 我们记 $G$ 在 $\phi$ 下的交叉数为 $c r_{\phi}(G)$. 更多关于交叉数方面的知识, 请读者参阅文献 [2] 及其 参考文献. 图 $G$ 和 $H$ 的 Cartesian 积图, 记作 $G \square H$, 其顶点集为 $V(G \square H)=V(G) \times V(H)$, 边集 $E(G \square H)=\left\{\left(x_{1}, y_{1}\right)\left(x_{2}, y_{2}\right) \mid x_{1}=x_{2}\right.$ 且 $y_{1} y_{2} \in E(H)$ 或者 $y_{1}=y_{2}$ 且 $\left.x_{1} x_{2} \in E(G)\right\}$.

图的交叉数研究是一个经典却又十分困难的问题 ${ }^{[2]}$. 事实上, 计算图的交叉数是一个 NP- 完全 问题 ${ }^{[3]}$. 目前, 已得到了一些特殊图类与路、圈以及星图的 Cartesian 积图的交叉数 ${ }^{[4-16]}$. 特别地, 借 助拉链积运算, Cartesian 积图 $K_{1, m} \square P_{n}{ }^{[4]}$ 和 $K_{2, m} \square P_{n}{ }^{[6]}$ 的交叉数最近被先后确定. 自然地, 我们希 
望进一步得到完全三部图与路的 Cartesian 积图的交叉数. Klešc 在文献 [7] 中确定了 $K_{1,1,3} \square P_{n}$ 的交 叉数. 本文进一步证明了: 对于 $m, n \geqslant 1$, 有

$$
\operatorname{cr}\left(K_{1,1, m} \square P_{n}\right)=2 n\left\lfloor\frac{m}{2}\right\rfloor\left\lfloor\frac{m-1}{2}\right\rfloor+(n-1)\left\lfloor\frac{m}{2}\right\rfloor .
$$

结论的证明基于 Bokal 文献 [5] 中的一个结果. 另外, 我们在第 4 节中也给出了确定 $K_{2, m} \square P_{n}$ 交叉数 的一个简洁方法.

\section{2 定义和引理}

设 $A$ 和 $B$ 是 $E$ 的两个互不相交的边子集. 在画法 $\phi$ 中, 用 $c r_{\phi}(A, B)$ 表示由集合 $A$ 中的边与 集合 $B$ 中的边产生的交叉个数, 用 $c r_{\phi}(A)$ 表示由集合 $A$ 中的边与边产生的交叉个数. 由交叉数的定 义, 如下命题显然成立.

命题 2.1 设 $\phi$ 是图 $G$ 的一个画法, $A, B, C$ 为 $E(G)$ 的互不相交的边子集. 则,

$$
\begin{aligned}
& c r_{\phi}(A \cup B)=c r_{\phi}(A)+c r_{\phi}(B)+c r_{\phi}(A, B), \\
& c r_{\phi}(A, B \cup C)=c r_{\phi}(A, B)+c r_{\phi}(A, C) .
\end{aligned}
$$

引理 2.1 (参见文献 [17]) 当 $m \leqslant 6$ 时, $c r\left(K_{m, n}\right)=\left\lfloor\frac{m}{2}\right\rfloor\left\lfloor\frac{m-1}{2}\right\rfloor\left\lfloor\frac{n}{2}\right\rfloor\left\lfloor\frac{n-1}{2}\right\rfloor$.

引理 2.2 当 $m \geqslant 1$ 时, $\operatorname{cr}\left(K_{1,2, m}\right)=\left\lfloor\frac{m}{2}\right\rfloor\left\lfloor\frac{m-1}{2}\right\rfloor$.

证明 $K_{1,2, m}$ 包含子图 $K_{3, m}$, 由引理 2.1 得 $\operatorname{cr}\left(K_{1,2, m}\right) \geqslant \operatorname{cr}\left(K_{3, m}\right)=\left\lfloor\frac{m}{2}\right\rfloor\left\lfloor\frac{m-1}{2}\right\rfloor$. 另一方面, 图 1 展示了一个含有 $\left\lfloor\frac{m}{2}\right\rfloor\left\lfloor\frac{m-1}{2}\right\rfloor$ 个交叉的 $K_{1,2, m}$ 画法. 因此, 引理得证.

引理 2.3 (参见文献 [18]) 当 $m \geqslant 1$ 时, $\operatorname{cr}\left(K_{1,3, m}\right)=2\left\lfloor\frac{m}{2}\right\rfloor\left\lfloor\frac{m-1}{2}\right\rfloor+\left\lfloor\frac{m}{2}\right\rfloor$.

为了方便表述与阅读, 下面我们重复文献 [5] 中的相关定义和结论.

设 $G_{i}, i=1,2$ 为图, $v_{i} \in V\left(G_{i}\right)$, 且 $\operatorname{deg}_{G_{i}}\left(v_{i}\right)=d$, 用 $N_{i}=N_{G_{i}}\left(v_{i}\right)$ 表示 $v_{i}$ 的邻域集. 称双射函 数 $\sigma: N_{1} \rightarrow N_{2}$ 为 $G_{1}$ 和 $G_{2}$ 在顶点 $v_{1}$ 和 $v_{2}$ 处的拉链函数. 在 $G_{1}-v_{1}$ 和 $G_{2}-v_{2}$ 的并图中添加边 $u \sigma(u), u \in N_{1}$, 得到的图称为 $G_{1}$ 和 $G_{2}$ 的拉链积, 记作 $G_{1} \odot_{\sigma} G_{2}$.

将图 $G$ 的所有顶点分别与 $i$ 个孤立点 $\left\{v_{1}, v_{2}, \ldots, v_{i}\right\}$ 相连, 得到的图称为是 $G$ 的 $i$ 阶悬挂, 记作 $G^{(i)}$, 其中 $v_{1}, v_{2}, \ldots, v_{i}$ 称为 $G^{(i)}$ 的尖端. 设 $L \subseteq V\left(G_{2}\right)$ 为多重集, 对于 $v \in L$ 的每个拷贝, 增加一个

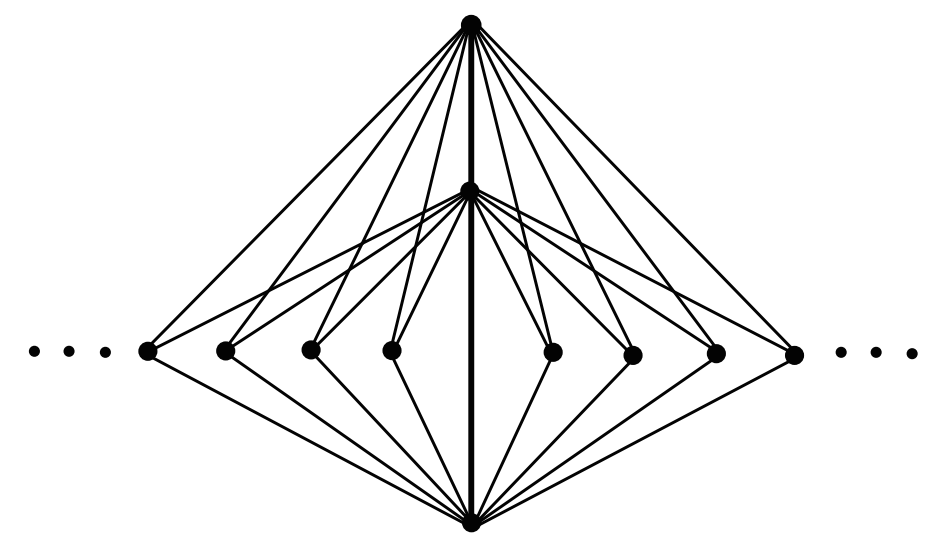

图 $1 K_{1,2, m}$ 的一个画法 
新的顶点 $v^{\prime}$, 并将 $v^{\prime}$ 与 $G_{1} \square\{v\}$ 的所有顶点相连, 这样得到的图称为 $G_{1}$ 与 $G_{2}$ 的带帽 Cartesian 积图, 记作 $G_{1} \square_{L} G_{2}$, 并称 $v^{\prime}$ 为 $v$ 的帽子. 对于 $v \in V\left(G_{2}\right)$, 用 $\chi_{L}(v)$ 表示 $v$ 在 $L$ 中的重数, 令 $\ell(v):=\operatorname{deg}_{G_{2}}(v)+\chi_{L}(v)$. 对于 $E\left(G_{2}\right)$ 中的边 $u v$, 如果 $\ell(u) \neq \ell(v)$, 则称 $u v$ 是不平衡的. 用 $\beta\left(G_{2}\right)$ 表 示 $G_{2}$ 中不平衡边的数目.

图 $G^{(i)}$ 的一个画法 $\phi$ 称为是尖端齐次的, 如果 $G$ 存在一个顶点置换 $\rho$, 使得每个尖端的顶点旋 系或者是 $\rho$ 或者是 $\rho^{-1} \cdot G^{(i)}$ 的画法 $\phi^{(i)}$ 与 $G^{(j)}$ 的画法 $\phi^{(j)}$ 称为是互相尖端齐次的, 如果 $\phi^{(i)}$ 和 $\phi^{(j)}$ 相应于同一个置换 $\rho$ 是尖端齐次的. 图 $G$ 有全尖端齐次画法, 如果存在 $G^{(i)}$ 的画法 $\phi^{(i)}, i \geqslant 1$, 使得 它们两两互相尖端齐次. 更多相关细节, 可参阅文献 $[4,5,19]$.

本文主要结果的证明基于 Bokal 的下述引理.

引理 2.4 (参见文献 [5]) 设 $G$ 为 $n$ 阶图, $T$ 是一棵树, $L \subseteq V(T)$ 是一个多重集, 且对于任意 $v \in V(T)$, 或者 $\ell(v) \geqslant 3$, 或者 $\ell(v) \geqslant 2$ (若 $G$ 含有一个支配点). 定义

$$
B=\sum_{v \in V(T)} c r\left(G^{(\ell(v))}\right),
$$

则, $B \leqslant \operatorname{cr}\left(G \square_{L} T\right) \leqslant B+\frac{\beta(T)}{2}\left(\begin{array}{l}n \\ 2\end{array}\right)$. 并且, 如果 $G$ 有全尖端齐次画法, 则 $\operatorname{cr}\left(G \square_{L} T\right)=B$.

\section{$3 \quad K_{1,1, m} \square P_{n}$ 的交叉数}

设 $\{a\},\{b\}$ 和 $Z=\left\{z_{1}, z_{2}, \ldots, z_{m}\right\}$ 为完全三部图 $K_{1,1, m}$ 的 3 个划分点集. 用 $\{0,1,2, \ldots, n\}$ 表示 $P_{n}$ 的顶点集, 且对于 $i=0,1,2, \ldots, n-1$, 点 $i$ 与点 $i+1$ 相邻. 为表述方便, 对于任意 $x \in Z \cup\{a, b\}$, 以及 $i=0,1, \ldots, n$, 用 $x^{i}$ 表示 $K_{1,1, m} \square P_{n}$ 中的顶点 $(x, i)$. 对于 $i=0,1, \ldots, n, j=0,1, \ldots, m+1$, 分 别用 $K_{1,1, m}^{i}$ 以及 $P_{n}^{j}$ 表示 $K_{1,1, m} \square P_{n}$ 中的 $n+1$ 个 $K_{1,1, m}$ 拷贝和 $m+2$ 个 $P_{n}$ 拷贝.

设 $G$ 为图, $\phi$ 是 $G$ 的一个画法, 对于 $v \in V(G), e \in E(G)$, 我们用 $r_{\phi}(v)$ 表示在画法 $\phi$ 下与顶点 $v$ 关联的所有边上产生的交叉个数, 用 $r_{\phi}(e)$ 表示在画法 $\phi$ 下边 $e$ 上产生的交叉个数.

引理 3.1 对于 $m, n \geqslant 1$, 有 $c r\left(K_{1,1, m} \square P_{n}\right) \leqslant 2 n\left\lfloor\frac{m}{2}\right\rfloor\left\lfloor\frac{m-1}{2}\right\rfloor+(n-1)\left\lfloor\frac{m}{2}\right\rfloor$.

证明 图 2 展示的是 $K_{1,1, m} \square P_{n}$ 的一个画法 $\phi$. 不难看出, 对于 $i, j=0,1, \ldots, n$ 且 $i \neq j$, 有 $c r_{\phi}\left(K_{1,1, m}^{i}\right)=0$ 以及 $c r_{\phi}\left(K_{1,1, m}^{i}, K_{1,1, m}^{j}\right)=0$.

对于 $i=1,2, \ldots, n-1$, 有

$$
\begin{aligned}
r_{\phi}\left(a^{i}\right) & =\sum_{j=1}^{\left\lfloor\frac{m}{2}\right\rfloor} r_{\phi}\left(a^{i} z_{j}^{i}\right)+\sum_{j=\left\lfloor\frac{m}{2}\right\rfloor+1}^{m} r_{\phi}\left(a^{i} z_{j}^{i}\right)+r_{\phi}\left(a^{i} b^{i}\right) \\
& =\left\lfloor\left(\left\lfloor\frac{m}{2}\right\rfloor-1\right)+\left(\left\lfloor\frac{m}{2}\right\rfloor-2\right)+\cdots+0\right\rfloor+\left[0+1+\cdots+\left(\left\lfloor\frac{m}{2}\right\rfloor-1\right)\right]+\left\lfloor\frac{m}{2}\right\rfloor \\
& =\left\lfloor\frac{m}{2}\right\rfloor\left\lfloor\frac{m-1}{2}\right\rfloor+\left\lfloor\frac{m}{2}\right\rfloor .
\end{aligned}
$$

类似地, 对于 $i=1,2, \ldots, n-1$, 有

$$
r_{\phi}\left(b^{i}\right)=\left\lfloor\frac{m}{2}\right\rfloor\left\lfloor\frac{m-1}{2}\right\rfloor+\left\lfloor\frac{m}{2}\right\rfloor .
$$

于是不难得到, 对于 $i=1,2, \ldots, n-1$,

$$
c r_{\phi}\left(K_{1,1, m}^{i}, \bigcup_{j=0}^{m+1} P_{n}^{j}\right)=\sum_{j=1}^{m}\left[r_{\phi}\left(a^{i} z_{j}^{i}\right)+r_{\phi}\left(b^{i} z_{j}^{i}\right)\right]+r_{\phi}\left(a^{i} b^{i}\right)
$$




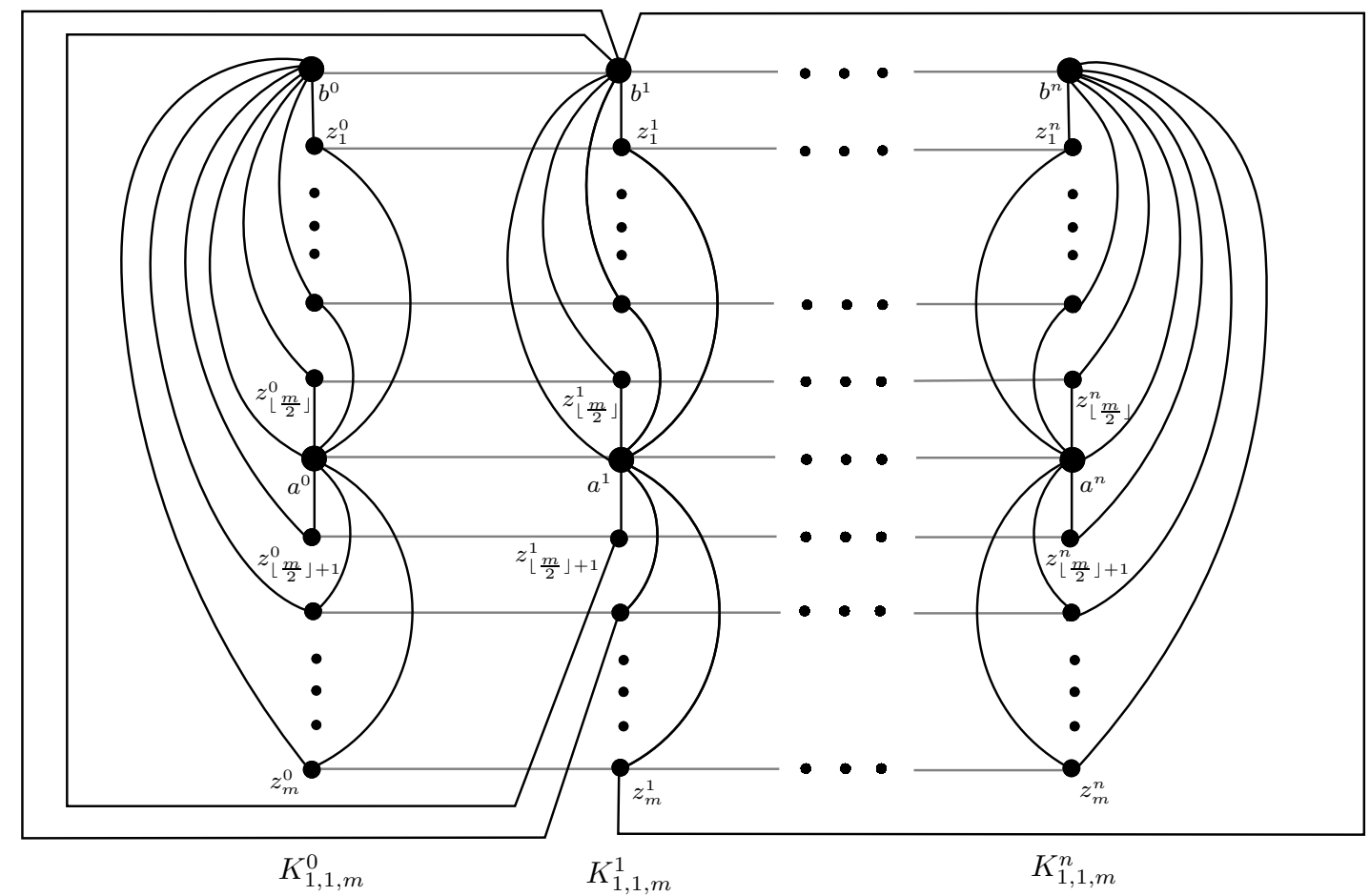

图 $2 K_{1,1, m} \square P_{n}$ 的一个画法 $\phi$

$$
\begin{aligned}
& =r_{\phi}\left(a^{i}\right)+r_{\phi}\left(b^{i}\right)-r_{\phi}\left(a^{i} b^{i}\right) \\
& =2\left\lfloor\frac{m}{2}\right\rfloor\left\lfloor\frac{m-1}{2}\right\rfloor+\left\lfloor\frac{m}{2}\right\rfloor .
\end{aligned}
$$

另外, 对于 $i=0, n$, 我们又有

$$
\begin{aligned}
r_{\phi}\left(a^{i}\right) & =\sum_{j=1}^{\left\lfloor\frac{m}{2}\right\rfloor} r_{\phi}\left(a^{i} z_{j}^{i}\right)+\sum_{j=\left\lfloor\frac{m}{2}\right\rfloor+1}^{m} r_{\phi}\left(a^{i} z_{j}^{i}\right)+r_{\phi}\left(a^{i} b^{i}\right) \\
& =\left\lfloor\left(\left\lfloor\frac{m}{2}\right\rfloor-1\right)+\left(\left\lfloor\frac{m}{2}\right\rfloor-2\right)+\cdots+0\right]+\left[0+1+\cdots+\left(\left\lceil\frac{m}{2}\right\rfloor-1\right)\right]+0 \\
& =\left\lfloor\frac{m}{2}\right\rfloor\left\lfloor\frac{m-1}{2}\right\rfloor
\end{aligned}
$$

并且 $c r_{\phi}\left(K_{1,1, m}^{i}, \bigcup_{j=0}^{m+1} P_{n}^{j}\right)=r_{\phi}\left(a^{i}\right)=\left\lfloor\frac{m}{2}\right\rfloor\left\lfloor\frac{m-1}{2}\right\rfloor$.

从而,

$$
\begin{aligned}
c r_{\phi}\left(K_{1,1, m} \square P_{n}\right) & =\sum_{i=0}^{n} c r_{\phi}\left(K_{1,1, m}^{i}, \bigcup_{j=0}^{m+1} P_{n}^{j}\right) \\
& =(n-1)\left(2\left\lfloor\frac{m}{2}\right\rfloor\left\lfloor\frac{m-1}{2}\right\rfloor+\left\lfloor\frac{m}{2}\right\rfloor\right)+2\left\lfloor\frac{m}{2}\right\rfloor\left\lfloor\frac{m-1}{2}\right\rfloor \\
& =2 n\left\lfloor\frac{m}{2}\right\rfloor\left\lfloor\frac{m-1}{2}\right\rfloor+(n-1)\left\lfloor\frac{m}{2}\right\rfloor .
\end{aligned}
$$


因此, 引理得证.

定理 3.1 对于 $m, n \geqslant 1$, 有

$$
\operatorname{cr}\left(K_{1,1, m} \square P_{n}\right)=2 n\left\lfloor\frac{m}{2}\right\rfloor\left\lfloor\frac{m-1}{2}\right\rfloor+(n-1)\left\lfloor\frac{m}{2}\right\rfloor .
$$

证明 根据引理 3.1, 我们只需证明

$$
\operatorname{cr}\left(K_{1,1, m} \square P_{n}\right) \geqslant 2 n\left\lfloor\frac{m}{2}\right\rfloor\left\lfloor\frac{m-1}{2}\right\rfloor+(n-1)\left\lfloor\frac{m}{2}\right\rfloor .
$$

设多重集 $L$ 包含路 $P_{n}$ 的所有顶点, 其中每个顶点的重数均为 1. 由引理 2.2-2.4 可以得到

$$
\begin{aligned}
\operatorname{cr}\left(K_{1, m} \square_{L} P_{n}\right) & \geqslant 2 \operatorname{cr}\left(K_{1, m}^{(2)}\right)+(n-1) \operatorname{cr}\left(K_{1, m}^{(3)}\right) \\
& =2 \operatorname{cr}\left(K_{1,2, m}\right)+(n-1) \operatorname{cr}\left(K_{1,3, m}\right) \\
& =2\left\lfloor\frac{m}{2}\right\rfloor\left\lfloor\frac{m-1}{2}\right\rfloor+(n-1)\left(2\left\lfloor\frac{m}{2}\right\rfloor\left\lfloor\frac{m-1}{2}\right\rfloor+\left\lfloor\frac{m}{2}\right\rfloor\right) \\
& =2 n\left\lfloor\frac{m}{2}\right\rfloor\left\lfloor\frac{m-1}{2}\right\rfloor+(n-1)\left\lfloor\frac{m}{2}\right\rfloor .
\end{aligned}
$$

同时, 不难验证 $K_{1,1, m} \square P_{n}$ 包含子图 $K_{1, m} \square_{L} P_{n}$. 因此 $\operatorname{cr}\left(K_{1,1, m} \square P_{n}\right) \geqslant 2 n\left\lfloor\frac{m}{2}\right\rfloor\left\lfloor\frac{m-1}{2}\right\rfloor+(n-$ 1) $\left\lfloor\frac{m}{2}\right\rfloor$, 定理得证.

\section{$4 \boldsymbol{K}_{2, m} \square \boldsymbol{P}_{n}$ 的交叉数}

唐玲等人 ${ }^{[6]}$ 和郑文萍等人 ${ }^{[16]}$ 分别用不同的方法独立确定了 $K_{2, m} \square P_{n}$ 的交叉数. 下面, 我们 将给出确定 $K_{2, m} \square P_{n}$ 交叉数的一个简洁方法.

定理 4.1 (参见文献 $[6,16]$ ) 对于 $m, n \geqslant 1$, 有

$$
\operatorname{cr}\left(K_{2, m} \square P_{n}\right)=2 n\left\lfloor\frac{m}{2}\right\rfloor\left\lfloor\frac{m-1}{2}\right\rfloor .
$$

证明 图 2 展示了 $K_{1,1, m} \square P_{n}$ 的一个画法, 且 $K_{1,1, m} \square P_{n}$ 在此画法下的交叉数为 $2 n\left\lfloor\frac{m}{2}\right\rfloor\left\lfloor\frac{m-1}{2}\right\rfloor$ $+(n-1)\left\lfloor\frac{m}{2}\right\rfloor$. 在此画法中, 去掉边 $a^{i} b^{i}, i=0,1, \ldots, n$, 可得到的 $K_{2, m} \square P_{n}$ 的一个画法, 并且在去边过 程中可以保证减少的交叉数为 $(n-1)\left\lfloor\frac{m}{2}\right\rfloor$. 因此 $\operatorname{cr}\left(K_{2, m} \square P_{n}\right) \leqslant 2 n\left\lfloor\frac{m}{2}\right\rfloor\left\lfloor\frac{m-1}{2}\right\rfloor$. 接下来我们只需证明

$$
\operatorname{cr}\left(K_{2, m} \square P_{n}\right) \geqslant 2 n\left\lfloor\frac{m}{2}\right\rfloor\left\lfloor\frac{m-1}{2}\right\rfloor .
$$

设多重集 $L$ 包含路 $P_{n}$ 的所有顶点, 其中每个顶点的重数均为 2 . 用 $\bar{K}_{m}$ 表示含有 $m$ 个顶点的 空图. 根据引理 2.1 和 2.4 , 有

$$
\begin{aligned}
\operatorname{cr}\left(\bar{K}_{m} \square_{L} P_{n}\right) & \geqslant 2 \operatorname{cr}\left(\bar{K}_{m}^{(3)}\right)+(n-1) \operatorname{cr}\left(\bar{K}_{m}^{(4)}\right) \\
& =2 \operatorname{cr}\left(K_{3, m}\right)+(n-1) \operatorname{cr}\left(K_{4, m}\right) \\
& =2\left\lfloor\frac{m}{2}\right\rfloor\left\lfloor\frac{m-1}{2}\right\rfloor+2(n-1)\left\lfloor\frac{m}{2}\right\rfloor\left\lfloor\frac{m-1}{2}\right\rfloor \\
& =2 n\left\lfloor\frac{m}{2}\right\rfloor\left\lfloor\frac{m-1}{2}\right\rfloor .
\end{aligned}
$$

同时, 不难验证 $K_{2, m} \square P_{n}$ 包含子图 $\bar{K}_{m} \square_{L} P_{n}$. 所以 $\operatorname{cr}\left(K_{2, m} \square P_{n}\right) \geqslant 2 n\left\lfloor\frac{m}{2}\right\rfloor\left\lfloor\frac{m-1}{2}\right\rfloor$, 定理得证. 致谢 诚挚地感谢审稿人和编委提出的宝贵意见和建议. 


\section{参考文献}

1 Bondy J A, Murty U S R. Graph Theory with Applications. London: Macmillan Press Ltd, 1976

2 Erdös P, Guy R K. Crossing number problems. Amer Math Monthly, 1973, 80: 52-58

3 Garey M R, Johnson D S. Crossing number is NP-complete. SIAM J Algeb Disc Meth, 1983, 4: 312-316

4 Bokal D. On the crossing number of Cartesian products with paths. J Combin Theory Ser B, 2007, 97: 381-384

5 Bokal D. On the crossing numbers of Cartesian products with trees. J Graph Theory, 2007, 56: 287-300

6 Tang L, Lv S X, Huang Y Q. The Crossing number of Cartesian products of complete bipartite graphs $K_{2, m}$ with paths $P_{n}$. Graphs Combin, 2007 23: 659-666

7 Klešč M. The crossing numbers of certain Cartesian products. Discuss Math Graph Theory, 1995, 15: 5-10

8 Klešč M. On the crossing numbers of products of stars and graphs of order five. Graphs Combin, 2001, 17: 289-294

9 Jendrol S, Ščerbová M. On the crossing numbers of $S_{m} \square P_{n}$ and $S_{m} \square C_{n}$. Čas Pest Mat, 1982, 107: 225-230

10 Glebsky L Y, Salazar G. The crossing number of $C_{m} \square C_{n}$ is as conjectured for $n \geqslant m(m+1)$. J Graph Theory, 2004, 47: $53-72$

11 Adamsson J, Richter R B. Arrangements, circular arrangements and the crossing number of $C_{7} \square C_{n}$. J Combin Theory Ser B, 2004, 90: 21-39

12 Salazar G. Drawings of $C_{m} \square C_{n}$ with one disjoint family. J Combin Theory Ser B, 1999, 76: 21-39

13 Shahrokhi F, Sýkora O, Székely L A, et al. Intersection of curves and crossing number of $C_{m} \square C_{n}$ on surfaces. Discrete Comput Geom, 1998, 19: 237-247

14 Ouyang Z D, Wang J, Huang Y Q. The crossing number of Cartesian product of paths with complete graphs. Discrete Math, 2014, 328: 71-78

15 Zheng W P, Lin X H, Yang Y S, et al. On the crossing number of $K_{m} \square P_{n}$. Graphs Combin, 2007, 23: 327-336

16 Zheng W P, Lin X H, Yang Y S. The crossing number of $K_{2, m} \square P_{n}$. Discrete Math, 2008, 308: 6639-6644

17 Kleitman D J. The crossing number of $K_{5, n}$. J Combin Theory Ser B, 1971, 9: 315-323

18 Asano K. The crossing number of $K_{1,3, n}$ and $K_{2,3, n}$. J Graph Theory, 1986, 10: 1-8

19 Beaudou L, Bokal D. On the sharpness of some results relating cuts and crossing numbers. Electron J Combin, 2010, 17: \#R96

\section{On the crossing number of $K_{1,1, \mathrm{~m}} \square \boldsymbol{P}_{n}$}

\section{OUYANG ZhangDong, WANG Jing \& HUANG YuanQiu}

Abstract The crossing numbers of the Cartesian products $K_{1, m} \square P_{n}$ and $K_{2, m} \square P_{n}$ were recently determined with the zip product operation. In this paper, we further show that

$$
\operatorname{cr}\left(K_{1,1, m} \square P_{n}\right)=2 n\left\lfloor\frac{m}{2}\right\rfloor\left\lfloor\frac{m-1}{2}\right\rfloor+(n-1)\left\lfloor\frac{m}{2}\right\rfloor
$$

for $n \geqslant 1, m \geqslant 1$. Our proof depends on Bokal's results for Cartesian products with trees. Furthermore, we give a simple proof for the crossing number of $K_{2, m} \square P_{n}$.

Keywords crossing number, Cartesian product, zip product, path

$\operatorname{MSC}(2010) \quad 05 \mathrm{C10}, 05 \mathrm{C} 62$

doi: $10.1360 / 012014-57$ 